\title{
Long-term outcome of pediatric non-cirrhotic portal fibrosis from the viewpoint of endoscopic profile
}

\author{
Kohei Kotani ${ }^{1} \cdot$ Norifumi Kawada $^{1}$
}

Received: 26 December 2019 / Accepted: 21 February 2020 / Published online: 13 March 2020

(c) Asian Pacific Association for the Study of the Liver 2020

Non-cirrhotic portal fibrosis (NCPF) presents with noncirrhotic portal hypertension characterized by moderate to severe splenomegaly, evidence of varices and/or collaterals, patent splenoportal axis and hepatic veins, normal liver functions, normal or near-normal hepatic venous pressure gradient (HVPG), and no evidence of cirrhosis or parenchymal injury in the liver histology [1]. The disease occurs worldwide but is common in socioeconomically disadvantaged countries, including India, and patients are generally young in their third and fourth decades of life. A similar disease group called idiopathic portal hypertension (IPH) in Japan has female preponderance and presents in the fourth and fifth decades of life [2]. This condition is known as idiopathic non-cirrhotic portal hypertension (INCPH) in the western countries [3].

The liver histology of NCPF has a wide spectrum ranging from obliterative sclerosis of portal vein branches and portal fibrosis to nodular regenerative hyperplasia. It is characterized by phlebosclerosis, fibroelastosis, periportal and perisinusoidal fibrosis, aberrant vessels in the portal tract (portal angiomatosis), preserved lobular architecture, and differential atrophy [4]. On the other hand, congenital hepatic fibrosis (CHF) in children is a differential disease. Most cases of CHF are associated with autosomal recessive disorder that affects the renal and hepatobiliary systems, and reveal presinusoidal portal hypertension and developmental abnormalities. CHF is characterized by diffuse periportal fibrosis. The fibrous bands surround single or multiple lobules. Numerous uniform small bile ducts and interrupted circular arrangement of the ducts can also be observed. The abnormality has been known as a ductal plate malformation within the fibrous band [4].

Norifumi Kawada

kawadanori@med.osaka-cu.ac.jp

1 Department of Hepatology, Graduate School of Medicine, Osaka City University, 1-4-3 Asahimachi, Abeno-ku, Osaka 545-8585, Japan
In Japan, research on intractable diseases and portal hemodynamics abnormalities, including IPH, has been conducted by the Ministry of Health, Labour and Welfare since 1975, and nationwide epidemiological surveys were performed in 1999, 2005, and 2015 [2]. In contrast to Japan, India had no recent nationwide survey on NCPF. However, recently, pediatric cases of NCPF have been frequently reported [5]. In the pediatric cohort, the disease remains unaware due to lack of subjective symptoms and often misdiagnosed as cryptogenic cirrhosis. Previously unnoticed NCPF cases may have now been detected owing to the development of medical resources. Therefore, it is important to understand the overall characteristics of adult NCPF patients and to investigate the natural history and prognosis of pediatric patients. In addition, the assessment of differences in clinical features between adult and pediatric patients of NCPF is desired.

In this issue of Hepatology International, Prasad et al. [6] comprehensively described the endoscopic management of pediatric patients with NCPF, especially the relationship between the natural history of esophageal varices and longterm outcome after treatment. Forty-five patients (3.3\%) aged $<18$ years who had portal hypertension were diagnosed as having NCPF. The median overall age at diagnosis and duration of symptoms was 14.5 years and 12 months, respectively. Of all patients, $78 \%$ had spleen-related issues; $96 \%$, esophageal varices; $56 \%$, primary gastric varices; and $89 \%$, portal hypertensive gastropathy. Almost $50 \%$ of patients (22 patients) presented with variceal bleeding and required a secondary prophylaxis. Of the 23 patients without variceal bleeding, 11 were treated endoscopically as primary prophylaxis. Moreover, of the 33 patients who underwent endoscopic treatment as primary or secondary prophylaxis, $36 \%$ showed recurrence of esophageal varices after a median follow-up of 11 months and 12\% developed secondary gastric varices. Overall, $87 \%$ of the patients required endoscopic interventions at onset or follow-up. Ten patients had poor outcomes, but the remaining $76 \%$ received regular 
endoscopic surveillance and remained asymptomatic, with generally favorable long-term outcomes.

Esophageal variceal bleeding is the common manifestation in adult patients with NCPF or IPH, but the prognosis is good if bleeding can be prevented [7, 8]. Few reports investigated the natural history of NCPF in pediatric patients [5]. This important study by Prasad et al. [6] evaluated the long-term outcome of pediatric patients with NCPF after endoscopic management. We should learn from the study that eradication of esophageal varices is associated with good prognosis also in pediatric patients with NCPF. Pediatric patients with NCPF would have a shorter morbidity period after the appearance of presinusoidal obliteration than adult patients, although this remains a speculation. Therefore, a good outcome can be expected unless variceal bleeding recurs, as the hepatic functional reserve is relatively maintained in pediatric patients unlike in adult patients. In a recent epidemiological study that followed up the longterm prognosis of patients with IPH for 10 years, 34\% of patients experienced gastrointestinal bleeding at the time of IPH diagnosis. In $11 \%$ of these patients, either the disease aggravated or the patient died during follow-up [2]. In the study by Prasad et al. [6], 49\% of patients experienced variceal bleeding at the time of NCPF diagnosis and 24\% had poor outcomes. Although the proportion of patients with poor outcomes was somewhat high, a simple comparison is difficult because of differences in age, living conditions, ethnicity, or medical facilities and systems between countries. Additional observational studies to analyze pediatric NCPF cohorts with longer follow-up periods are desired.

According to the APASL consensus statement [1], endoscopic variceal ligation therapy is the first recommendation for treatment of large varices in NCPF patients. The use of beta-blockers in such patients may be effective, but no consensus has been obtained. In this study by Prasad et al. [6], there was no difference in the recurrence rate of esophageal and large gastric varices with or without beta-blockers administration. Balloon-occluded retrograde transvenous obliteration may be an option in patients with large gastric varices. On the other hand, there is insufficient data on the role of transjugular intrahepatic portosystemic shunt although it is expected to be useful for reduction of portal pressure. The therapeutic effect of surgery on NCPF remains controversial. Splenectomy, or non-selective or selective shunt surgery is recommended for patients with marked splenomegaly who tend to have bleeding with low platelet counts that is difficult to control with endoscopic procedures. Liver transplantation is required for patients with intractable ascites, hepatopulmonary syndrome, or liver failure [4, 8]. Experience is abundant in surgical procedures for extrahepatic portal vein obstruction, but data on NCPF are limited. Splenectomy and shunt surgery can reduce portal vein pressure but confer the risk of developing hepatic encephalopathy, ascites, and portal thrombus [4]. In this study by Prasad et al. [6], three patients were decompensated with cirrhosis and referred for liver transplantation and four underwent surgery (one for emergency splenectomy and three for splenorenal shunt procedure with splenectomy). Of the three patients who underwent shunt surgery, none had encephalopathy and none died during follow-up. Although the mid-term therapeutic effect of surgery is good, further studies are expected to assess the longer-term outcome.

The main study limitation was that portal vein pressure or HVPG was not measured. Therefore, the relationship between esophageal variceal bleeding or long-term outcome and portal pressure cannot be confirmed even if patients with NCPF often exhibit near-normal portal vein pressure. Moreover, the exact time of onset of NCPF is difficult to determine, and the disease duration could be underestimated. Thus, whether the true disease duration of NCPF contributes to esophageal variceal bleeding is unclear. In NCPF, several environmental chemicals, drugs, and organic compounds are considered as causes. Arsenic is most frequently described, followed by vinyl chloride, copper sulfate, methotrexate, 6-mercaptopurin, azathioprine, busulfan, didanosine, vita$\min \mathrm{A}$, and so on. According to the review by Khanna et al. [4], hydroxyproline and collagen levels increased without significant hepatic fibrosis in the chronic arsenic exposure animal model. Furthermore, in IPH or INCPH, several reports have indicated that immune abnormalities, including human immunodeficiency virus infection, splenic dysfunction, and abnormal coagulopathy, are involved in the pathogenesis $[3,9,10]$. Whether the long-term outcome of pediatric NCPF after intervention with endoscopic management is associated with these environmental factors, immune abnormalities, splenic dysfunction, and/or coagulation abnormalities remain to be elucidated (Fig. 1). Further studies are warranted to evaluate these issues.

In summary, the work by Prasad et al. [6] reports the relationship between the endoscopic management and longterm outcome of pediatric patients with NCPF. Patients with and without bleeding had similar outcomes, and careful endoscopic surveillance is important. Some patients with bleeding had poor outcome or required surgical procedures, but most pediatric patients with NCPF retained an adequate hepatic functional reserve and a good prognosis. In the future, a long-term prognostic epidemiological survey will be conducted for 10 years, depending on the variceal status in pediatric patients with NCPF, and the prognostic improvement effect of endoscopic intervention will be further evaluated. 
Fig. 1 Future perspective on long-term prognostic assessment in patients with pediatric NCPF. Further surveys will be conducted to investigate the relationship between endoscopic management or variceal bleeding and PVP or HVPG, true disease duration, treatment options, environmental factors, immune abnormalities, splenic dysfunction, and coagulation abnormalities. NCPF noncirrhotic portal fibrosis, $P V P$ portal vein pressure, $H V P G$ hepatic venous pressure gradient, TIPS transjugular intrahepatic portosystemic shunt

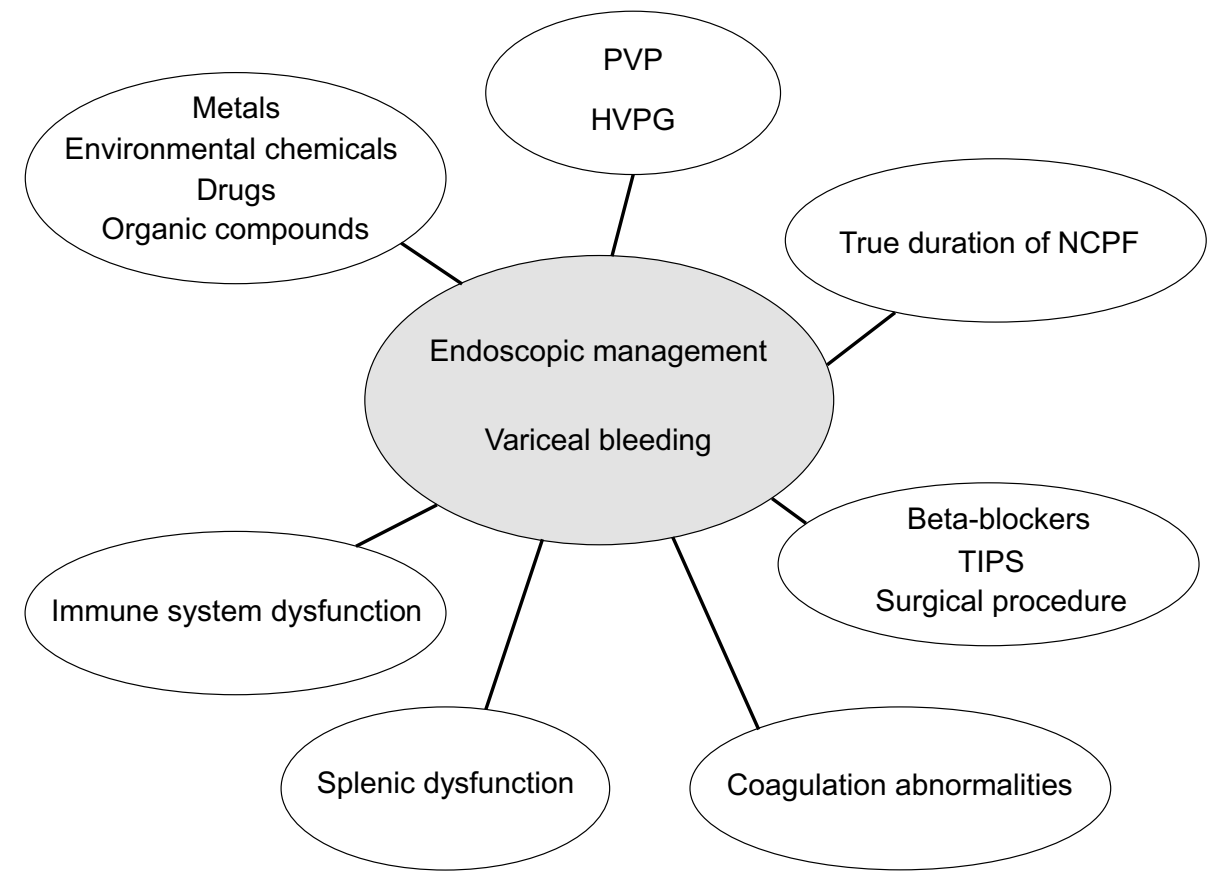

Funding None.

\section{Compliance with ethical standards}

Conflict of interest Kohei Kotani and Norifumi Kawada declare that they have no conflict of interest regarding this article.

\section{References}

1. Sarin SK, Kumar A, Chawla YK, Baijal SS, Dhiman RK, Jafri W, et al. Noncirrhotic portal fibrosis/idiopathic portal hypertension: APASL recommendations for diagnosis and treatment. Hepatol Int 2007;1:398-413

2. Ohfuji S, Furuichi Y, Akahoshi T, Kage M, Obara K, Hashizume $\mathrm{M}$, et al. Japanese periodical nationwide epidemiologic survey of aberrant portal hemodynamics. Hepatol Res 2019;49:890-901

3. Schouten JN, Garcia-Pagan JC, Valla DC, Janssen HL. Idiopathic noncirrhotic portal hypertension. Hepatology 2011;54:1071-1081

4. Khanna R, Sarin SK. Non-cirrhotic portal hypertension - diagnosis and management. J Hepatol 2014;60:421-441

5. Sood V, Lal BB, Khanna R, Rawat D, Bihari C, Alam S. Noncirrhotic portal fibrosis in pediatric population. J Pediatr Gastroenterol Nutr 2017;64:748-753
6. Prasad D, Sen Sarma M, Yachha SK, Srivastava A, Poddar U. Pediatric non-cirrhotic portal fibrosis: role of endoscopic management in determining long-term outcome. Hepatol Int 2019 (Epub ahead of print)

7. Dhiman RK, Chawla Y, Vasishta RK, Kakkar N, Dilawari JB, Trehan MS, et al. Non-cirrhotic portal fibrosis (idiopathic portal hypertension): experience with 151 patients and a review of the literature. J Gastroenterol Hepatol 2002;17:6-16

8. Siramolpiwat S, Seijo S, Miquel R, Berzigotti A, Garcia-Criado A, Damell A, et al. Idiopathic portal hypertension: natural history and long-term outcome. Hepatology 2014;59:2276-2785

9. Kotani K, Kawabe J, Morikawa H, Akahoshi T, Hashizume M, Shiomi S. Comprehensive screening of gene function and networks by DNA microarray analysis in Japanese patients with idiopathic portal hypertension. Mediators Inflamm 2015;2015:349215

10. Dabritz J, Worch J, Materna U, Koch B, Koehler G, Duck C, et al. Life-threatening hypersplenism due to idiopathic portal hypertension in early childhood: case report and review of the literature. BMC Gastroenterol 2010;10:122

Publisher's Note Springer Nature remains neutral with regard to jurisdictional claims in published maps and institutional affiliations. 